Research Article

\title{
Combined Integrating Control Based on Dynamic Optimization Estimation
}

\author{
Angang Chen, ${ }^{1,2}$ Zhengyun Ren $\mathbb{D}^{1},{ }^{1}$ Zhiping Fan, ${ }^{1,2,3}$ and Xue Feng ${ }^{1}$ \\ ${ }^{1}$ College of Information Science and Technology, Donghua University, Shanghai 201620, China \\ ${ }^{2}$ Department of Chemical and Materials Engineering, University of Alberta, Edmonton, AB T6G 2G6, Canada \\ ${ }^{3}$ School of Electrical and Electronic Engineering, Anhui Science and Technology University, Fengyang 233100, China
}

Correspondence should be addressed to Zhengyun Ren; renzhengyun@dhu.edu.cn

Received 23 April 2020; Revised 4 June 2020; Accepted 13 June 2020; Published 10 July 2020

Academic Editor: Eric Florentin

Copyright (C) 2020 Angang Chen et al. This is an open access article distributed under the Creative Commons Attribution License, which permits unrestricted use, distribution, and reproduction in any medium, provided the original work is properly cited.

In this paper, a new type of controller based on the combined integrating systems is proposed, which is called a combined integrating controller. The controller's current output is determined only by the current input and the average value of the controller's previous output. The innovation is to introduce a time-delay positive feedback in the controller. The memory characteristics of the time-delay term keep accumulating error information and repeatedly learning so that the system can stably track and restrain any input signal without error. Simultaneously, the combined integrating controller is applied to conventional process systems based on dynamic optimization estimation in the case study to show absolute superiority over the nonpredictive control method (such as the classical PID control method).

\section{Introduction}

The Proportional-Integral-Derivative (PID) control algorithms and their variants are widely used in the process industry because they can meet most control requirements for various processes. Most PID tuning rules are based on conventional process models in the industry, such as the first-order process with time-delay (FOPDT), second-order process with time-delay (SOPDT), integrating processes, and high-order processes. The widespread use of FOPDT models, especially in PID controllers, is due to their simplicity and ability to capture the essential dynamics of multiple industrial processes [1-3]. On the other hand, many industrial processes can be successfully approximated by a second-order time-delay transfer function. Especially in the chemical process industry, most control loops are PID-type second-order modeling plants or temperature control, as shown in $[4,5]$. Compared to stable process models, the number of PID adjustment rules reported for the integrating process is less in the literature. The integrating process is challenging to control because load disturbances easily disrupt the balance between the input and output. In [6], controllers of the modified Smith predictor are designed for pure integrating, integrating plus first order and double integrating processes with large time-delay. However, if there is a larger time-delay in the process, the control performance obtained using the PID controller will be limited [7]. It may cause the dynamic performance of the control system to decline or the stability of the entire system to deteriorate and even cause system oscillation or divergence. Numerous methods of PID controller synthesis and tuning have been developed over the past few decades, such as Ziegler-Nicholas based tuning methods [8], internal model control (IMC) design methods $[9,10]$, frequencydomain method [11-13], and decomposition method based on multi-scale control (MSC) scheme $[14,15]$.

With the in-depth research on the process industry model, many complex processes may not be able to adequately describe with conventional models, such as fractional integral and differential express the dynamics of the actual process more flexible and accurate [16]. The distillation column bottom process, which Morari had referred to in [17], has a transfer function $(k / s)\left(1-2 e^{-\theta_{1} s}\right) e^{-\theta_{2} s}$, which is a nonminimum phase time-delay process. Morari 
designed the controller of this kind of process based on the IMC and verified the control performance.

At the same time, through the analysis of the mechanism of the productive process, we get a new type of system transfer function that contains the $\left(1-e^{-\theta s}\right) / \theta s$ term and call it a combined integrating system. This kind of system has been widely used in the fields of steel, chemical industry, and tobacco through our engineering practice. The most combined integrating systems are regarded as a low-order timedelay (LOTD) using the conventional control strategies, such as the PID control algorithm, Smith predictor algorithm, or IMC algorithm. To some extent, these control algorithms satisfy the needs of industrial production, which is why these processes are not taken seriously.

Nevertheless, with the rapid development of modern industry, product quality, energy consumption, and environmental protection put higher requirements on precision control. The traditional control has gradually been not suitable for combined integrating systems. Therefore, it needs to design new control strategies and methods to satisfy higher requirements. In this paper, a new type of controller based on the combined integrating systems is proposed, which is called a combined integrating controller. The purpose is to provide a dead-time compensating controller that can handle multi-time-delay systems and manually adjust control parameters in the same way as PID controllers.

In the classical control period, the time-delay term is often regarded as a fixed part of the system, to be stabilized rather than as a control parameter. In [18], the time-delay term is introduced into the controller, and the time-delay coefficient is taken as the control parameter. In this paper, the time-delay and the combined integrating term are introduced into the controller and as control parameters. The effective combination of time-delay term and combined integrating term with "slow computing" characteristics is used to construct a controller with a simple structure and reduced computational complexity. In the following simulation, we will give the theoretical feasibility. The advantages of introducing the time-delay term and combined integrating term as control parameters in the control system are as follows: (1) the controller is simple, and the parameters depend on the time-delay and combined integrating term of the model. (2) The controller's current output is only determined by the current input and the average value of the controller's previous output. (3) The asynchronous operation of the control system is realized, which is easily converted into a digital controller, similar to a hybrid system.

This paper is organized as follows. In Section 2, dynamic modeling and estimation are introduced. In Section 3, based on the internal model principle and predictive control, we propose a combined integrating controller and give a detailed derivation process. In Section 4, the combined integrating controller mechanism analysis is introduced. In Section 5, the combined integrating controller robustness analysis is presented. In Section 6, we will compare the different control algorithms for the typical industrial processes based on dynamic optimization estimation. Finally, we give conclusions and further study in Section 7.

\section{Dynamic Modeling and Estimation}

The critical aspect of any controller is getting a sufficiently accurate model. The model form can include adjustable parameters that cannot be directly measured but can be adjusted to match steady-state and dynamic data. Models can be linear or nonlinear, empirical, or based on primary forms produced by materials and energy balances, reaction kinetics mechanisms, or other predefined model structures. The model structure can also include constraints such as fixed gain ratio, constraints on components, or other boundaries reflecting physical reality. Equation (1) is a statement of the general model form, which can include differential, algebraic, continuous, and integer variables:

$$
\begin{aligned}
\operatorname{minimize} \quad J(x, y, p, d, u), & \\
\text { s.t. } \quad 0 & =f\left(\frac{\mathrm{d} x}{\mathrm{~d} t}, x, y, p, d, u\right), \\
0 & =g_{1}(x, y, p, d, u), \\
0 & \leq g_{2}(x, y, p, d, u), \\
x(0) & =x_{0} .
\end{aligned}
$$

The target value of equation (1) is determined by the initial state $x_{0}$, a set of parameters $p$, a trajectory of disturbance values $d$, and a sequence of control moves $u$. The equation includes differential $f$, algebraic $g_{1}$, and inequality constraints $g_{2}$ that are included to model physical phenomena. In addition to the model form, the objective function $J$ is vital to ensure desirable results. A common objective form is a least-squares form: $\left(y_{\text {model }}-y_{\text {measured }}\right)^{2}$. A suitable initial guess for the state trajectories is often required for solver convergence.

The direct application of DAE to nonlinear optimization faces many challenges. In this paper, it is assumed that the dynamic model can be described by a set of ordinary differential-algebraic equations (DAEs). The discretization of continuous-time representation allows large-scale nonlinear programming (NLP) solvers to find solutions at specified intervals over time. There are many names and related techniques to find the mathematical relationship between the derivative and non-derivative. These techniques include orthogonal collocation on finite elements, direct transcription, Gauss pseudospectral method, Gaussian quadrature, Lobatto quadrature, Radau collocation, Legendre polynomials, Chebyshev polynomials, Jacobi polynomials, Laguerre polynomials, and so on [19-23]. Here, we use the orthogonal collocation method on the finite element with Lobatto quadrature to solve the DAEs (the differential term in equation (2)):

$$
\frac{\mathrm{d} x}{\mathrm{~d} t}=h(x, y, p) .
$$

Euler's method is based on two-node per horizon step linear discretization by 


$$
\begin{aligned}
\frac{\mathrm{d} x}{\mathrm{~d} t} & =\frac{x(k+1)-x(k)}{\Delta T}=h(x, y, p), \\
x(k+1) & =h(x, y, p) \Delta T+x(k) .
\end{aligned}
$$

The orthogonal collocation method on the finite element with Lobatto quadrature uses nonlinear polynomial fitting. In engineering applications, each horizontal step can be linear within 3-6 nodes. Here, we give four nodes orthogonally by Lobatto quadrature: $t_{0}=0, t_{1}=(1 / 2)-(\sqrt{5} / 10)$, $t_{2}=(1 / 2)+(\sqrt{5} / 10), t_{3}=1$ :

$$
\begin{gathered}
x(t)=A+B t+C t^{2}+D t^{3}, \\
\frac{\mathrm{d} x}{\mathrm{~d} t}=B+2 C t+3 D t^{2} .
\end{gathered}
$$

In this derivation, a third-order polynomial approximates the solution at the four points on the horizon. The increase in the number of collocation points will increase the corresponding polynomial order. For the initial value problem, when the initial time is arbitrarily defined as zero, the coefficient $A$ is equal to $x_{0}$. In order to determine the coefficients $B, C$, and $D$, equation (4) is substituted into equation (5), and the results are obtained. Note that the coefficient $A$ in equation (4) is offset by $x_{0}$ to the right of equation (5). Finally, the rearrangement and solution of $M$ give the solutions of the three nodes in the future which are given as follows:

$$
\begin{aligned}
& {\left[\begin{array}{l}
\frac{\mathrm{d} x_{1}}{\mathrm{~d} t} \\
\frac{\mathrm{d} x_{2}}{\mathrm{~d} t} \\
\frac{\mathrm{d} x_{3}}{\mathrm{~d} t}
\end{array}\right]=M\left(\left[\begin{array}{l}
x_{1} \\
x_{2} \\
x_{3}
\end{array}\right]-\left[\begin{array}{l}
x_{0} \\
x_{0} \\
x_{0}
\end{array}\right]\right),} \\
& {\left[\begin{array}{ccc}
1 & 2 t_{1} & 3 t_{1}^{2} \\
1 & 2 t_{2} & 3 t_{2}^{2} \\
1 & 2 t_{3} & 3 t_{3}^{2}
\end{array}\right]\left[\begin{array}{l}
B \\
C \\
D
\end{array}\right]=M\left[\begin{array}{lll}
t_{1} & t_{1}^{2} & t_{1}^{3} \\
t_{2} & t_{2}^{2} & t_{2}^{3} \\
t_{3} & t_{3}^{2} & t_{3}^{3}
\end{array}\right]\left[\begin{array}{l}
B \\
C \\
D
\end{array}\right],} \\
& M=\left[\begin{array}{lll}
1 & 2 t_{1} & 3 t_{1}^{2} \\
1 & 2 t_{2} & 3 t_{2}^{2} \\
1 & 2 t_{3} & 3 t_{3}^{2}
\end{array}\right]\left[\begin{array}{lll}
t_{1} & t_{1}^{2} & t_{1}^{3} \\
t_{2} & t_{2}^{2} & t_{2}^{3} \\
t_{3} & t_{3}^{2} & t_{3}^{3}
\end{array}\right]^{-1}, \\
& {\left[\begin{array}{l}
x_{1} \\
x_{2} \\
x_{3}
\end{array}\right]=M^{-1} h(x, y, p)+\left[\begin{array}{l}
x_{0} \\
x_{0} \\
x_{0}
\end{array}\right] \text {. }}
\end{aligned}
$$

A simultaneous solution demonstrates the application of orthogonal collocation. The first-order system $\tau(\mathrm{d} x / \mathrm{d} t)=$ $-x+k u$ is solved at $3-5$ points from $t_{0}=0.0$ to $t_{n}=5.0$ using orthogonal collocation on finite elements with Lobatto quadrature. In this case, $\tau=5.0, k=2.0$, and the initial condition is specified at $x_{0}=0.0$. For this problem, the time points for $\mathrm{d} x / \mathrm{d} t$ and $x$ are selected by Lobatto quadrature. The value of $x$ is specified at $t_{0}=0.0$ due to the initial condition. As shown in Figure 1, as the number of collocation points increases, the accuracy increases and the relative error decreases.

\section{Combined Integrating Systems and Combined Integrating Controller}

Definition 1. The system transfer function consists of a combined integral term, integrating plus time-delay process, and a general process system transfer function. It can be expressed as the following general equation:

$$
G_{p}(s)=\frac{k}{\theta_{1} s} G(s)\left(1-e^{-\theta_{1} s}\right) e^{-\theta_{2} s} .
$$

We define these open-loop stable systems as combined integrating systems. The combined integrating term $\left(1-e^{-\theta_{1} s}\right) / \theta_{1} s$ is an essential part of equation $(6) ; G(s)$ is a general process system transfer function without integrating. In recent years, it is found that four typical controlled processes are widely used in the industry. Their transfer functions are

$$
\begin{aligned}
& G_{p}(s)=\frac{k}{\theta_{1} s}\left(1-e^{-\theta_{1} s}\right), \\
& G_{p}(s)=\frac{k}{\theta_{1} s}\left(1-e^{-\theta_{1} s}\right) e^{-\theta_{2} s}, \\
& G_{p}(s)=\frac{k}{\theta_{1} s(\tau s+1)}\left(1-e^{-\theta_{1} s}\right), \\
& G_{p}(s)=\frac{k}{\theta_{1} s(\tau s+1)}\left(1-e^{-\theta_{1} s}\right) e^{-\theta_{2} s} .
\end{aligned}
$$

In the industrial process, the first-order Padé approximation is often used for the time-delay. So we use this approximate method for the combined integrating systems, and the combined integrating systems are transformed into the conventional industrial systems (LODT). However, its characteristics also have changed. The combined integrating systems are non-minimum phase systems, and the approximation results are transformed into minimum phase systems. The corresponding controllers are designed by the traditional control method; though it can get the control performance satisfying the production requirements, it is difficult to improve the control performance further.

Due to the specific characteristics of the combined integrating systems, using the traditional PID algorithm, IMC, prediction PI (PPI) algorithm, and Smith predictive control algorithm cannot reach good control effect, rapidity, and stability. It needs to design a new control algorithm. The controller design of the combined integrating systems (7a) and $(7 \mathrm{~b})$ is analyzed below. 


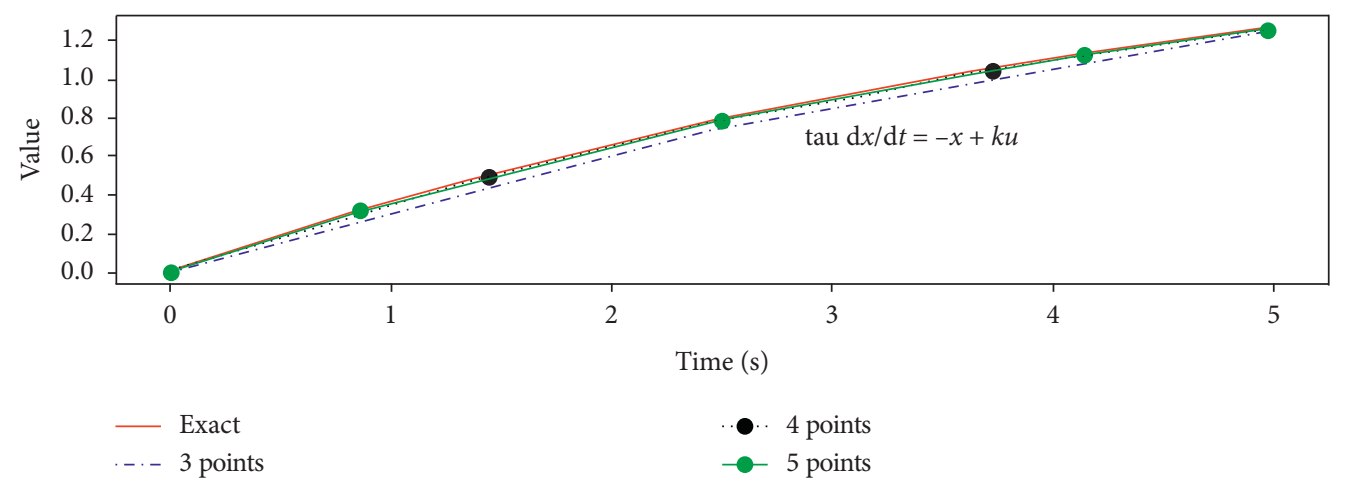

(a)

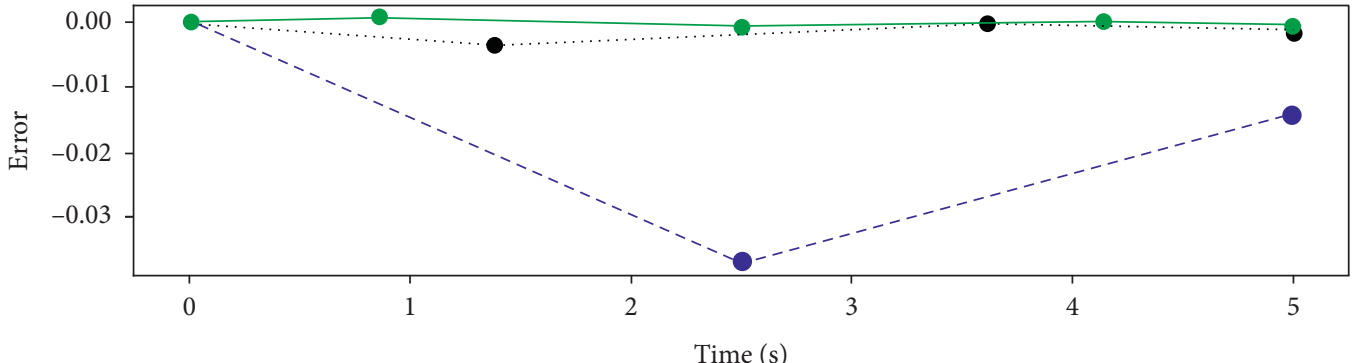

- 3 points
- 4 points
- 5 points

(b)

Figure 1: The orthogonal collocation method on the finite element with Lobatto quadrature to solve the DAEs at 3-5 points.

\section{Combined Integrating System (7a)}

By choosing the appropriate desired closed-loop response (see equation (8)) and using either a Padé approximation or a power-series approximation for the time-delay, PI/PID controllers can be derived for process models commonly used in industrial applications [24, 25]:

$$
G_{0}(s)=\frac{1}{0.5 \theta_{1} s+1} \text {. }
$$

According to the internal model controller's design principle, a two-degree-of-freedom controller is obtained, which can achieve the setpoint tracking performance, and the transfer function of the controller is

$$
G_{c}=\frac{U(s)}{E(s)}=\frac{1}{0.5 k\left(1-e^{-\theta_{1} s}\right)} \text {. }
$$

The relationship between the input $E(s)$ and the output $U(s)$ of the controller is

$$
U(s)=\frac{2}{k} E(s)+e^{-\theta_{1} s} U(s) .
$$

Equation (10) interprets that the current output of the new controller is determined only by the current input and previous controller output (see Figure 2).

The controller itself is a time-delay positive feedback controller, and its structure and realization are convenient and straightforward. The controller has good anti-disturbance

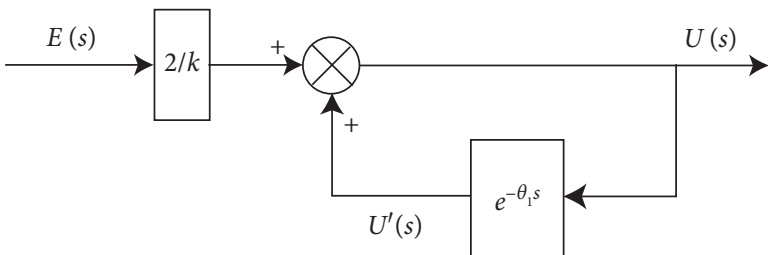

FIgURE 2: The original structure of the controller.

ability, response speed, and robustness. However, after the industrial application of the controller, it is found that the output oscillation of the controller is relatively large, and the wear of the actuator is relatively large, which severely limits its popularization in the industry.

To overcome the shortcomings of the controller output oscillation, it needs to smooth the positive feedback control part. Considering the combined integrating term $\left(1-e^{-\theta_{1} s}\right) / \theta_{1} s$ average filter nature, the time-delay $e^{-\theta_{1} s}$ in Figure 3 is replaced and the $2 / k$ is reduced to $1 / k$, so we get the following improvement of the controller structure.

In Figure 3, $U^{\prime}(s)$ is the Laplace transform of the average value of output $u(t)$ in the prior period $\left[t-\theta_{1}, t\right]$; the relationship between input and output of the controller in Figure 3 is

$$
U(s)=\frac{1}{k} E(s)+\frac{1}{\theta_{1} s}\left(1-e^{-\theta_{1} s}\right) U(s) .
$$




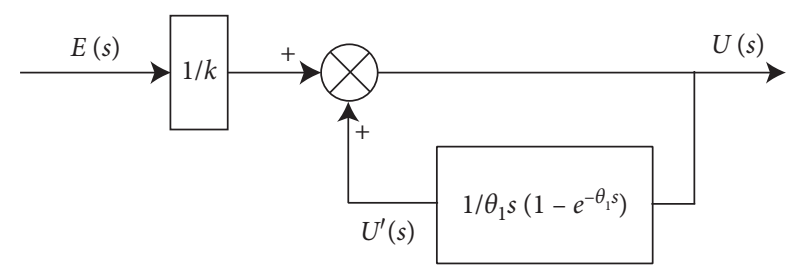

FIGURE 3: The improvement of the structure of the controller.

The transfer function of the controller is

$$
G_{c}=\frac{U(s)}{E(s)}=\frac{\theta_{1} s}{k\left(\theta_{1} s-1+e^{-\theta_{1} s}\right)} .
$$

The closed-loop transfer function of the whole system is

$$
G_{0}(s)=\frac{1}{\theta_{1} s}\left(1-e^{-\theta_{1} s}\right) .
$$

The closed-loop transfer function is consistent with the open-loop transfer function. Assuming discrete time is $T_{0}$, $t=n T_{0}, \theta_{1}=r T_{0}, n$ and $r$ are integers. Considering the convenience of implementation, transform equation (11) into an incremental form:

$$
\Delta u(n)=\frac{T_{0}}{k}[e(n)-e(n-1)]+\frac{T_{0}}{\theta_{1}}\left[u(n)-u\left(n-r_{1}\right)\right] .
$$

As can be seen from equation (14), the increment of controller output relates only to the current and previous inputs as well as the controller output in the prior period $\left[n-r_{1}, n\right]$.

\section{Combined Integrating System (7b)}

The system (7b) has a pure time-delay more than the first system, following the front controller design ideas. The current controlled variable is only related to the controller output in the prior period $\left[t-\theta_{1}-\theta_{2}, t-\theta_{2}\right]$. The controller's positive feedback term $\left(1-e^{-\theta_{1} s}\right) / \theta_{1} s$ is replaced by $\left(1-e^{-\theta_{1} s}\right) e^{-\theta_{2} s} / \theta_{1} s$, and the following controller structure is obtained, as shown in Figure 4.

The relationship between the input $E(s)$ and the output $U(s)$ of the controller is

$$
U(s)=\frac{1}{k} E(s)+\frac{1}{\theta_{1} s}\left(1-e^{-\theta_{1} s}\right) e^{-\theta_{2} s} U(s) .
$$

The input and output of equation (15) are discretized and transformed into an incremental form (assuming discretetime is $T_{0}, t=n T_{0}, \theta_{1}=r_{1} T_{0}, \theta_{2}=r_{2} T_{0}, n, r_{1}$ and $r_{2}$ are integers):

$$
\Delta u(n)=\frac{T_{0}}{k}[e(n)-e(n-1)]+\frac{T_{0}}{\theta_{1}}\left[u\left(n-r_{1}\right)-u\left(n-r_{1}-r_{2}\right)\right] .
$$

According to the above derivation, the definition of the combined integrating controller is a class controller with the combined integrating term $\left(1-e^{-\theta s}\right) / \theta s$, the controller output by the current input and the average value of previous controller output.

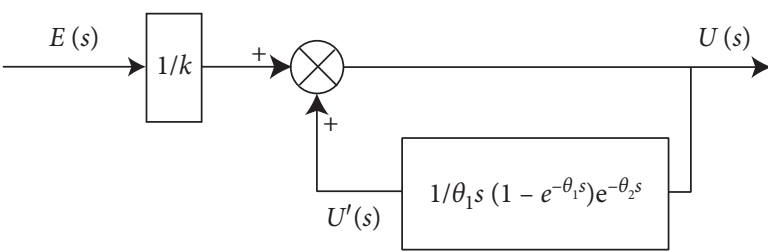

FIgure 4: Combined integrating controller structure.

Table 1 lists the combined integrating controllers of various types of combined integrating systems.

\section{Combined Integrating Controller Structure Analysis}

From equation (15) and Table 1, it can be seen that combined integrating controller structure contains a combined integrating term $\left(1-e^{-\theta_{1} s}\right) / \theta_{1} s$ and a time-delay term. The time-delay always is regarded as an unfavorable factor. More and more researchers show that the impact on time-delay on system performance is not always negative. By judiciously introducing time-delay into the system, the system performance and stability can be improved [26], which translates the unfavorable time-delay into a favorable one.

The current output of the combined integrating controller consists of the current input and the average controller output over the prior period. Due to the existence of $\left(1-e^{-\theta_{1} s}\right) / \theta_{1} s$, the combined integrating controller has the predictive function and eliminates the system steady-state residual error function. According to the definition of equation (15), we give the controller's specific parameters, that is, $U(s)=E(s)+(1 / 10 s)\left(1-e^{-10 s}\right) e^{-10 s} U(s)$, and simulate the step response of the controller (at $t=10 \mathrm{~s}$ plus step input). The controller response curve can be obtained, as shown in Figure 5.

From Figure 5, the output of the combined integrating controller consists of three parts: proportional action, predictive action (anti-delay), and integral action. Compared with the output of the traditional PID controller, there is an essential difference. Firstly, the prediction function is different. If the controller's input remains the same, the predictive effect will keep the controller unchanged in the pure time-delay. Secondly, the integral action is different; the strength of the integral is different at different periods. The most crucial point is that there is a different mechanism for achieving a steady-state residual error: the PID is achieved by direct integration of the inputs, and the combined integrating controller is obtained by averaging the output over a period. When the input remains constant, the average output value of the controller increases continuously due to the positive feedback structure of the combined integrating controller, to eliminate the steady-state residual.

\section{Combined Integrating Controller Robustness Analysis}

Robustness is the ability of the closed-loop system to be insensitive to component changes. It is one of the most useful properties of feedback. The presence of large time- 
TABLE 1: Combined integrating controllers for combined integrating systems.

\begin{tabular}{lcr}
\hline Process & Controller structure & Setting parameter \\
\hline$(7 \mathrm{a})$ & $\theta_{10} s / k_{0}\left(\theta_{10} s-1+e^{-\theta_{10} s}\right)$ & $k_{0}, \theta_{10}$ \\
$(7 \mathrm{~b})$ & $\theta_{10} s / k_{0}\left(\theta_{10} s-e^{\left(-\theta_{20} s\right)}+e^{-\left(\theta_{10}+\theta_{20}\right) s}\right)$ & $k_{0}, \theta_{10}, \theta_{20}$ \\
$(7 \mathrm{c})$ & $\theta_{10} s(\tau s+1) / k_{0}\left(\theta_{10} s(\tau s+1)-1+e^{-\theta_{10} s}\right)$ & $k_{0}, \theta_{10}, \tau_{10}$ \\
$(7 \mathrm{~d})$ & $\theta_{10} s\left(\tau_{10} s+1\right) / k_{0}\left(\theta_{10} s\left(\tau_{10} s+1\right)-e^{\left(-\theta_{20} s\right)}+e^{-\left(\theta_{10}+\theta_{20}\right) s}\right)$ & $k_{0}, \theta_{10}, \theta_{20}, \tau_{10}$ \\
\hline
\end{tabular}

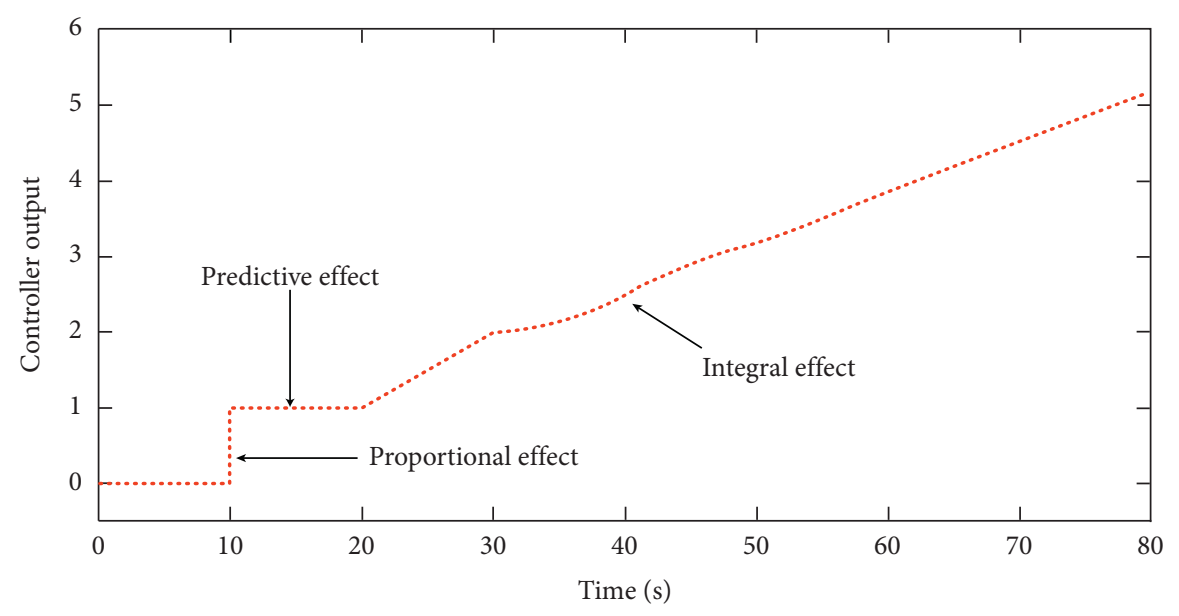

...... Controller response curve

FIGURE 5: Combined integrating controller's step response.

delay and parameter uncertainties in the process model poses a severe challenge to the design of robust feedback controls for chemical processes [27]. It is well known that a well-designed control system should meet the following requirements besides nominal stability: disturbance attenuation, setpoint tracking, robust stability, and robust performance [28-30].

The trade-off between stability and steady-state tracking accuracy is an important aspect of the performance of the combined integrating system. The stability analysis of the control system with a combined integrating controller is essentially stability analysis of a class of systems with multitime-delay. In recent years, many researchers have published relevant results and concluded that the stability space of multi-time-delay processes is discontinuous [29]. It is methodologically feasible to apply these research results to the stability research of the subject [31]. Such systems are time-delay systems with $\left(1-e^{-\theta s}\right) / \theta s$, which bring great difficulties to the stability analysis of control systems. Zheng $[32,33]$ uses the stability analysis method based on Kronecker's theory and Routh array to analyze the multi-timedelay system with coefficients and delay uncertainties.

The research of robust stability does not need to obtain the mathematical expression of stability. However, it needs to get the relationship between the stability and the controller parameters and provides the theoretical basis for the design and parameter tuning of the combined integrating controller. Therefore, to control more applications in the actual industry, we use the most practical industrial field debugging method to analyze the relative stability.
From Figure 6, the closed-loop system characteristic equation is as follows:

$$
D(s)=1+k \frac{1-e^{-\theta_{1} s}}{\theta_{1} s}-k \frac{1-e^{-\theta^{*} s}}{\theta^{*} s}=0,
$$

where $k, \theta_{1}$, and $\theta^{*}$ are all positive.

Theorem 1. Assume $f(x)=\left(1-e^{-x}\right) / x$ and $f(x)$ is a monotone decreasing function at $x>0$.

However,

$$
f(x)=\frac{1-e^{-x}}{x}, \quad f^{\prime}(x)<0 .
$$

Then, if $\theta^{*}>\theta_{1},\left(1-e^{-\theta_{1} s} / \theta_{1} s\right)-\left(1-e^{-\theta^{*} s} / \theta^{*} s\right)>0$. Assume $s=a+j b$, and then only $a<0$ can satisfy $D(s)=0$. Therefore, the relative stability is obtained by setting the range of controller parameter $\theta^{*}$; then, we get the more accurate range by the relevant approximation:

$$
\begin{aligned}
D(s) & =1+k \frac{1-e^{-\theta_{1} s}}{\theta_{1} s}-k \frac{1-e^{-\theta^{*} s}}{\theta^{*} s} \\
& =1+k \frac{1}{\theta_{1} s}\left(1-\frac{1-\theta_{1} s}{1+\theta_{1} s}\right)-k \frac{1}{\theta^{*} s}\left(1-\frac{1-\theta^{*} s}{1+\theta^{*} s}\right) \\
& =\theta_{1} \theta^{*} s^{2}+\left[(2 k+1) \theta^{*}-(2 k-1) \theta^{*}\right] s+1 .
\end{aligned}
$$

According to Routh array stability, 


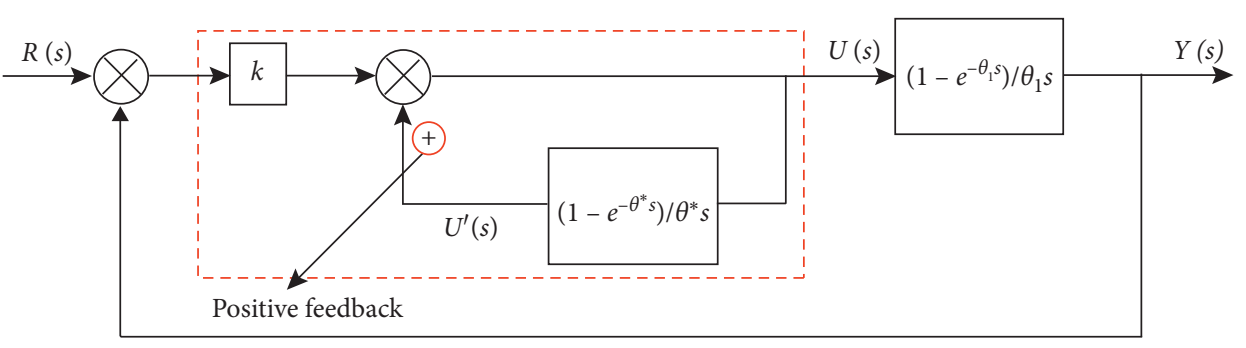

FIgURE 6: Combined integrating control block diagram.

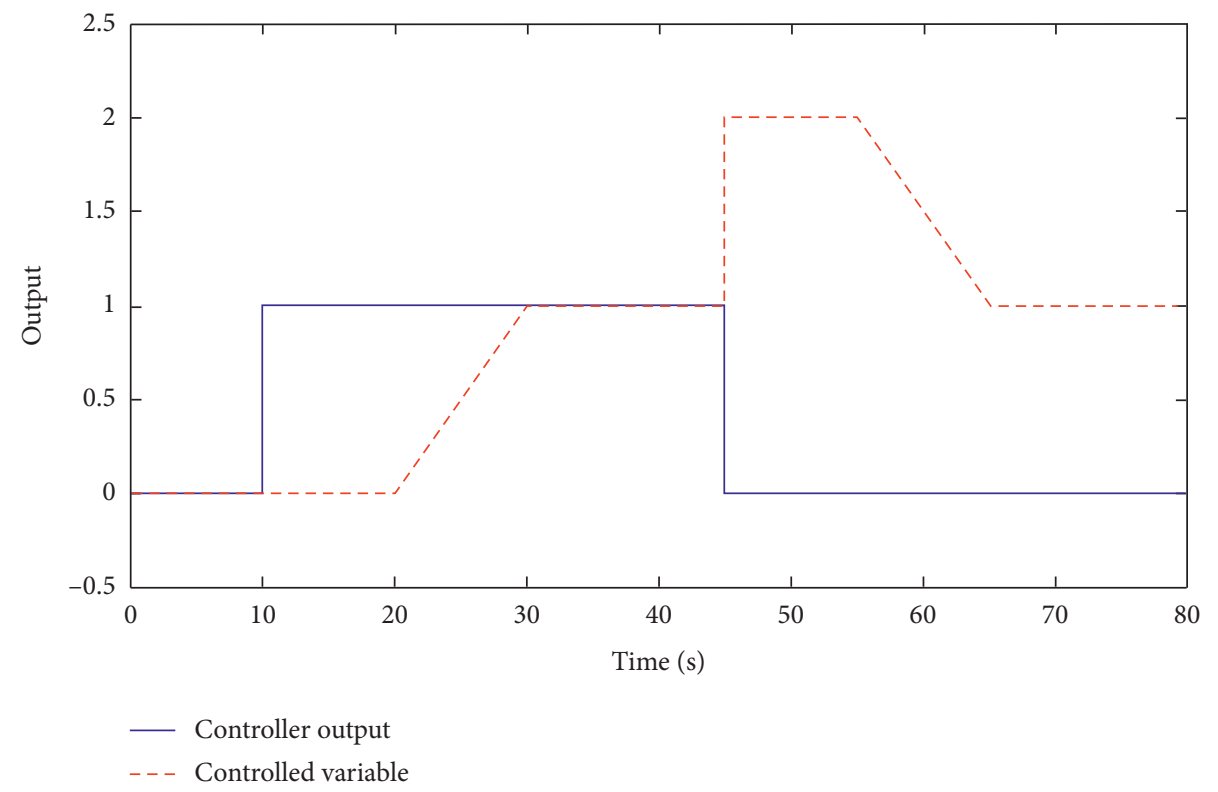

FIgURE 7: Control system step response and anti-jam response (no model mismatch).

$$
\theta^{*}>\frac{2 k-1}{2 k+1} \theta_{1}
$$

The combined integrating controller is used to stabilize the feedback system, which can achieve relative stability and a certain degree of parameter tuning. However, the robust stability of the combined integrating controller will be illustrated by the concrete simulation examples.

Assume that the parameters of the combined integrating system (7B) are as follows: $k=1, \theta_{1}=10$, and $\theta_{2}=10$, which is a kind of large time-delay process, using equation (15) to control. The step response curve and anti-jamming characteristics are shown in Figure 7 with a step increase at $t=10 \mathrm{~s}$ and a disturbance amplitude increase 1 at $t=45 \mathrm{~s}$ ).

When the model of controlled process is mismatched, the parameters are $k=1.1, \theta_{1}=11$, and $\theta_{2}=9$. While the controller remains the same, the system can still be well controlled as shown in Figure 8.

For the model structure mismatch of controlled process, it changes to the first-order large-time-delay process $G_{p}(s)=$ $(1 /(5 s+1)) e^{-10 s}$ (with a step increase at $t=10 \mathrm{~s}$ and a disturbance amplitude increase 1 at $t=100 \mathrm{~s}$ ). Similarly, the controller remains unchanged, the response speed of the system slows down to a certain extent, and the smoothness is lacking, but the system still has excellent control performance: there are no overshoot and no steady-state error and antijamming performance, and the controller output is relatively smooth, as shown in Figure 9.

\section{Case Study}

Above, we just verify that the proposed controller has excellent control characteristics for the combined integrating systems. Next, we compare it with PID and Predictive PI control algorithms for common industrial processes to verify the effectiveness of the control algorithm. Various performance indicators were selected for testing and comparison (e.g., response time, settling time, overshoot, and integral error (ITAE)) [28-30, 34].

In the modern sense, cigarette products' production process roughly goes through seven major production processes: tobacco leaf pre-curing, tobacco leaf re-curing, tobacco leaf fermentation, cigarette formula, cigarette shreds, cigarette rolls, and cigarette packaging. The tobacco leaf pre-curing cannot be directly supplied to the cigarette factory but must be re-cured, that is, the second moisture adjustment of the tobacco leaf based on the tobacco leaf precuring, making it a real raw material for cigarette production. Before the raw tobacco is re-cured, the tobacco slices and the stems are separated by the leaf-beating device. Tobacco leaf and stems were re-baked separately, packaged, 


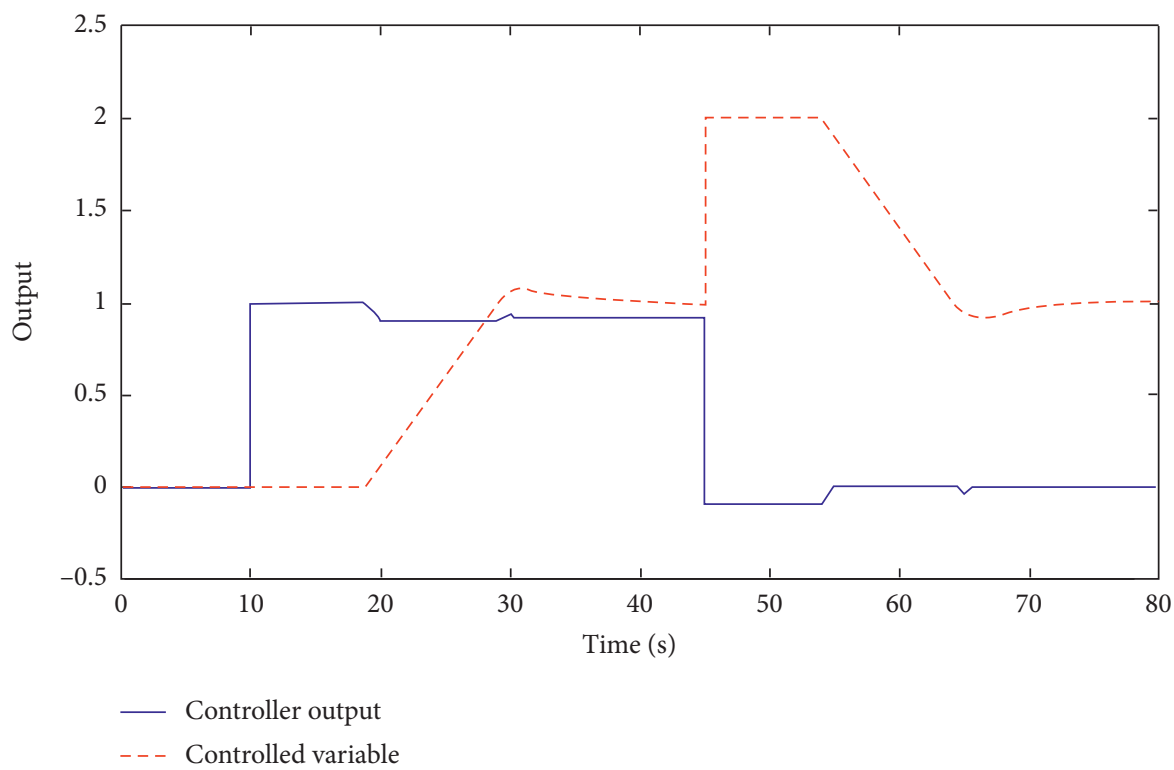

FIGURE 8: Control system step response and antijam response (model mismatch).

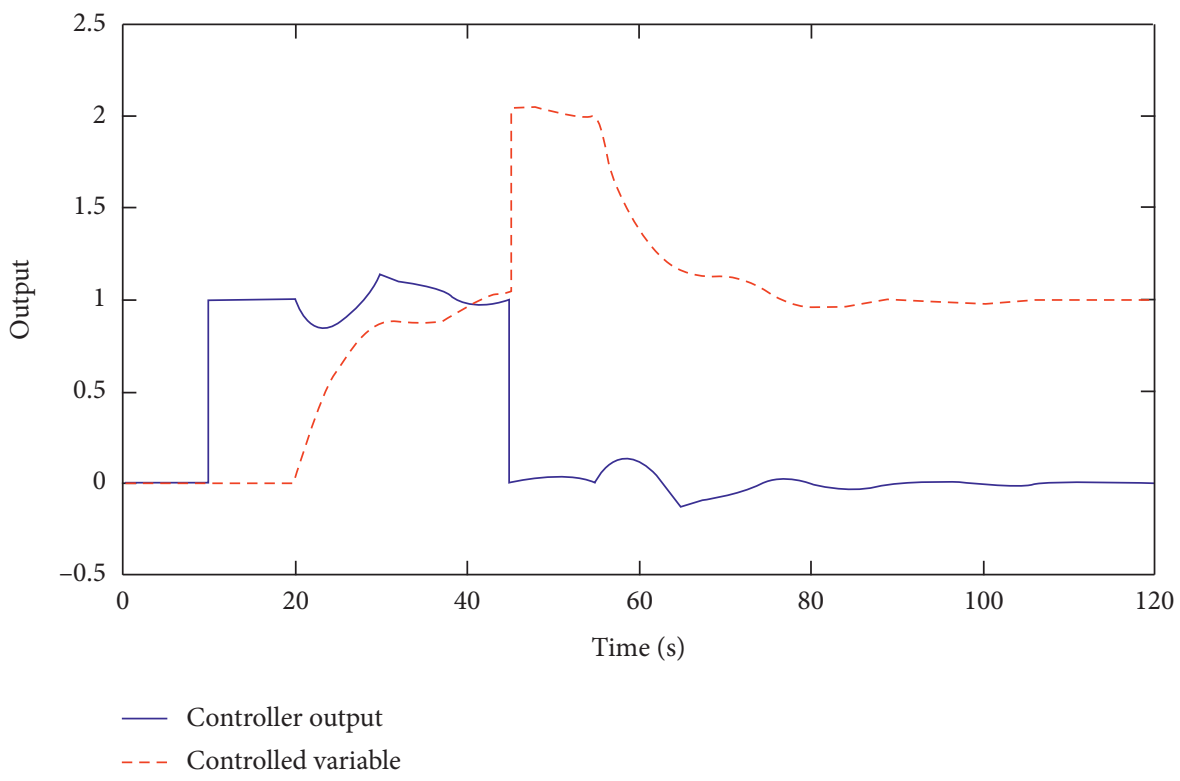

FIGURE 9: Control system step response and anti-jam response (model structure change).

and stored separately. Tobacco leaf pre-curing has uneven moisture content during grading and binding, and the climate and storage conditions are different in different regions.

The role of tobacco leaf re-curing: first, adjust the moisture to prevent mildew; second, remove impurities and purify aroma; third, insecticide and sterilization and beneficial storage; fourth, maintain color, and facilitate production. After reheating and drying, the physical and chemical characteristics of the re-cured tobacco leaf are further optimized. The quality of the tobacco leaf is improved, and the hygroscopicity is weakened, which is conducive to the natural alcoholization of the tobacco leaf during storage. To meet the requirements of threshing and reduce the shattering during threshing, it is necessary to increase the temperature and humidity of the tobacco leaf before threshing to improve the toughness and processing resistance of the tobacco leaf.

WF3 series hot-air leaf moisturizing machine needs to carry out secondary tobacco leaf moisture processing on the tobacco leaf after the moisture regaining machine so that the tobacco leaf's moisture increases from about $12 \%$ to $16 \%$ after the vacuum moisture regains, and the temperature reaches about $65^{\circ}$. The main body of the hot air leaf moisturizing machine is a slanting slewing body. Driven by the power device during operation, the cylinder continuously rotates, and the tobacco leaves first enter the drum from the upstream vibrating conveyor. On the inner surface 
of the drum, there are spirally distributed stirring rods. The rotation of the drum, the action of the stirring rod, and the tobacco leaf make a spiral movement with the cylinder. The drum rotates round and round, and the tobacco leaves are gradually sent out of the drum outlet.

During the flow of tobacco leaf in the cylinder, due to the relatively sealed state of the cylinder, the air jets from the three nozzles of steam, water-steam mixer, and hot air at the inlet form a high-temperature and high-humidity mixed airflow that quickly fills the relatively tight cylinder space, thus forming a high temperature and high humidity environment in the cylinder. The tobacco leaves are in full contact with the high-temperature and high-humidity steam and heated by the ambient humid and hot gas and absorb the moisture in the humid airflow. The heat and mass transfer between the tobacco leaf and the humid and hot gas causes the tobacco leaf to heat up and absorb moisture quickly. Under the joint suction action of the moisture discharge fan and the circulation fan at the outlet end of the drum, the hot and humid airflow in the drum flows from the inlet end to the outlet end of the drum. Figure 10 shows valves control the inlet flow of mixed water vapor and hot air. MT1 and MT3 are moisture sensor and transmitter from imported and exported tobacco leaf, FT2 is the flow sensor and transmitter of hot air, and FT1 is the flow sensor and transmitter of mixed water vapor.

Most of the tuning techniques are proposed for FOPDT. This paper presents the technique for obtaining the FOPDT model using dynamic optimization estimation. Most process engineers know how to adjust the different parameters of the controller to obtain desired closed-loop behaviors. They also know how the three parameters $k_{p}, T_{i}$, and $T_{d}$ influence the control. The empirical model building aims to find a relationship between the response (output) variable $y$ and the input $u$.

The controller requires an estimate of the static process gain, the dominating time constant, and the dead time. As seen above, these parameters are easily obtained from a step response experiment. Graphical method to obtain the static process gain, the apparent dead time, and the dominating time constant from a step response is given in Figure 11. However, this graphical method can only get an imprecise result. For the high-precision control system, it is impossible to use this identification method. Therefore, we choose to use the above dynamic optimization estimation method, at the same time, based on the results of the graphical method, as the initial value of dynamic optimization estimation, to increase the convergence and solution speed of optimization.

We get the system process model and input pressure disturbance model. The optimization parameters are as shown in Table 2.

In traditional control, there are two control methods for disturbance suppression: feedforward \& feedback and cascade control. Cascade control is one of the most popular complex control structures that can be found in the process industries, implemented to improve the disturbance rejection properties of the controlled system $[9,10,35]$. As shown in Figure 12, the system's goal is to control the outlet moisture of the tobacco leaf. The manipulated variable is the flow rate of the mixed water vapor, and the disturbance

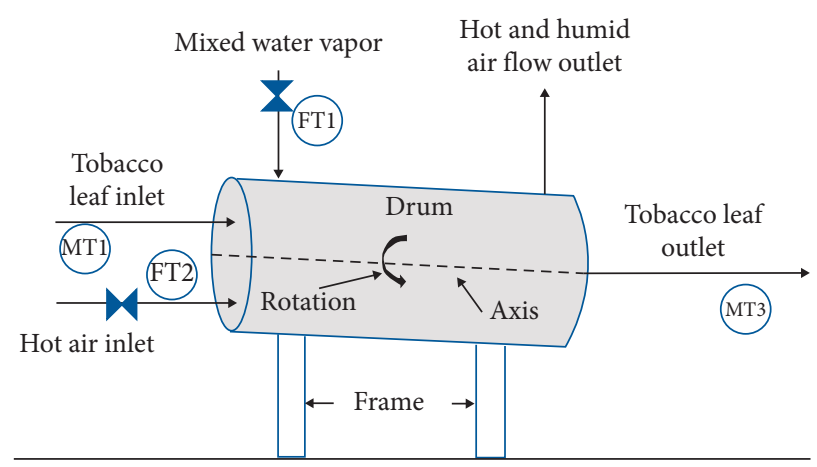

FIGURE 10: Scheme of the WF3 series hot-air leaf moisturizing machine.

variable is the inlet pressure of the mixed water vapor. MC3 is the primary controller controlling the outlet moisture of tobacco leaf, and PC is the sub-controller controlling the mixed water vapor inlet pressure. PT is the inlet pressure sensor and transmitter of mixed water vapor. Here, we use cascade control with the combined integrating controller (CIC), PPI, IMC PID method, Ziegler-Nichols method, and Lambda method, respectively, in Figure 12. We compare the performance of the five algorithms based on three situations.

8.1. Case 1. For the nominal process model and disturbance model that we have established, we control the process object through the combined integrating control (CIC), PPI, IMC PID, Ziegler-Nichols method, and Lambda method. From the setpoint tracking response in Figure 13, we can see that the CIC, PPI, and IMC PID control algorithms reach the setpoint value within 2000 seconds, while the ZieglerNichols and Lambda control algorithms fail to track the setpoint value, a meaningless control algorithm. From Table 3 , the overall performance indexes of the three effective control algorithms are counted.

The CIC control algorithm proposed in this paper is superior to PPI and IMC in terms of response time dynamic performance index and steady-state performance index (rise time, settling time, and steady-state error). The ITAE performance index from the system error also confirms that the CIC control performance is improved twice as much as the PPI and IMC.

8.2. Case 2. As another disturbance of the system, the hot air flow affects the moisture at the tobacco leaf outlet. When the hot air flow fluctuates positively, it will enhance the tobacco leaf humidification process and increase the tobacco leaf outlet moisture. We assume that, at $t=2000 \mathrm{~s}$, a disturbance model is added. The input of the first scheme is a signal with a positive step amplitude of 1, accompanied by Gaussian random noise (the mean of 0 , the variance of 0.5 ). The input of the second scheme is a signal with a negative step amplitude of 1, accompanied by Gaussian random noise (the mean of 0 , the variance of 0.5 ).

From the setpoint tracking response in Figure 14, we can see that the CIC, PPI, and IMC PID control algorithms 

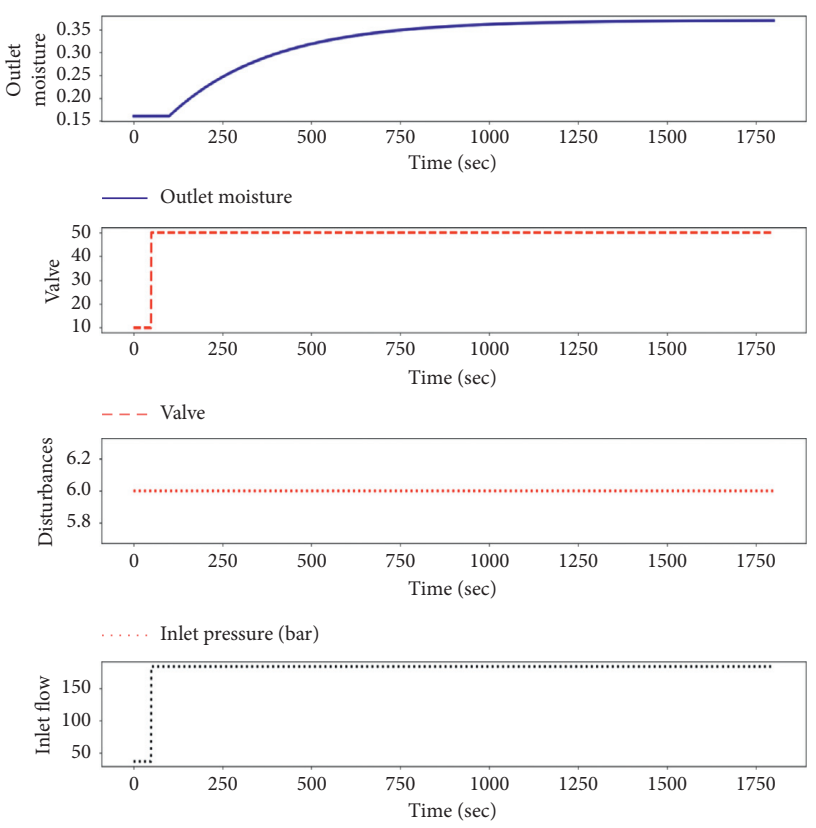

Inlet flow $(\mathrm{kg} / \mathrm{sec})$

(a)
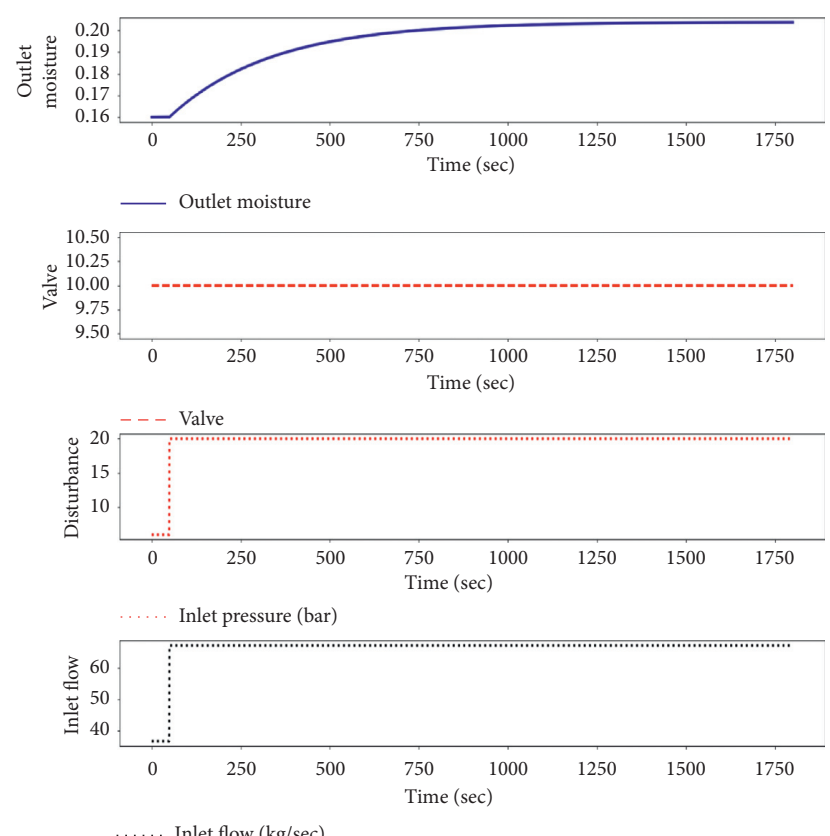

(c)

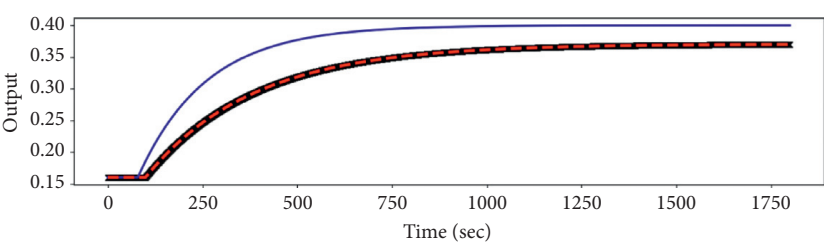

$\longrightarrow$ Process data

_ Initial guess

- - - Optimized FOPDT

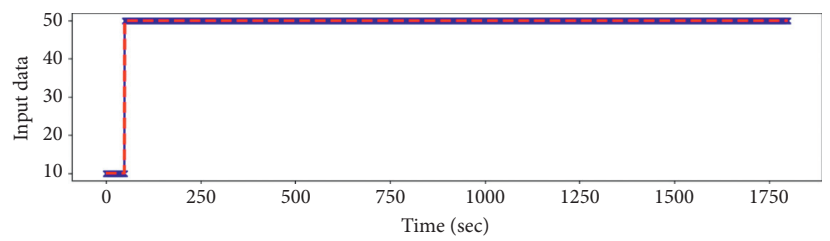

— Measured

- - Interpolated (b)

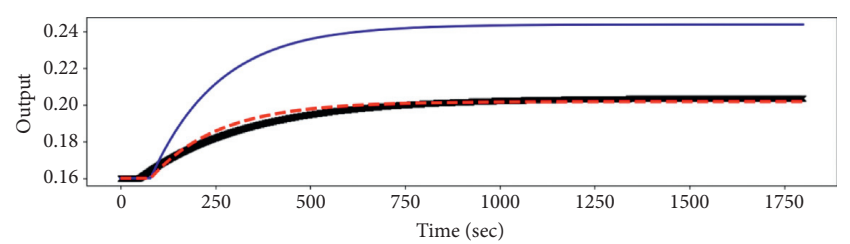

* Process data

_ Initial guess

- - - Optimized FOPDT

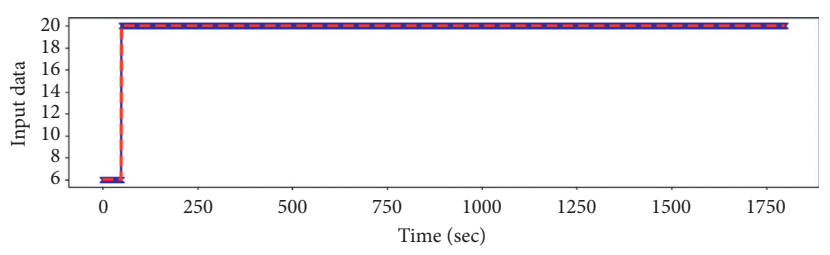

$\longrightarrow$ Measured

- - - Interpolated

Figure 11: FOPDT dynamic optimization estimation. (a) System process model step response. (b) System process model dynamic optimization estimation. (c) Pressure disturbance model step response. (d) Pressure disturbance model dynamic optimization estimation.

TABLE 2: The optimization parameters.

System process model

Initial SSE objective: 3.252622599256132

Final SSE objective: $7.48282342403205 e-07$

$k_{p}=0.005261136020866821$

$\tau_{p}=285.7114734241374$

$\begin{aligned} \tau_{p} & =285.7114734241374 \\ \theta_{p} & =50.30611754428681\end{aligned}$
Pressure disturbance model

Initial SSE objective: 2.591413522995586

Final SSE objective: 0.007572297982182136

$$
\begin{gathered}
k_{d}=0.0029858210411095707 \\
\tau_{d}=180.00000001004034 \\
\theta_{d}=30.00000210667573
\end{gathered}
$$




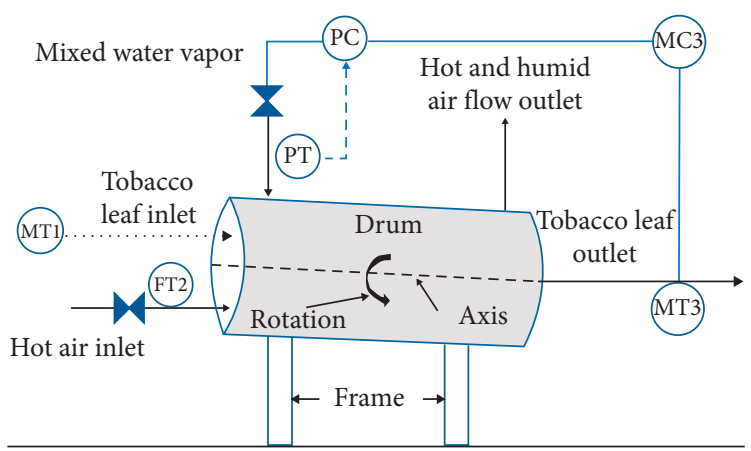

FIgURE 12: Cascade control block diagram of the WF3 series hot-air leaf moisturizing machine.

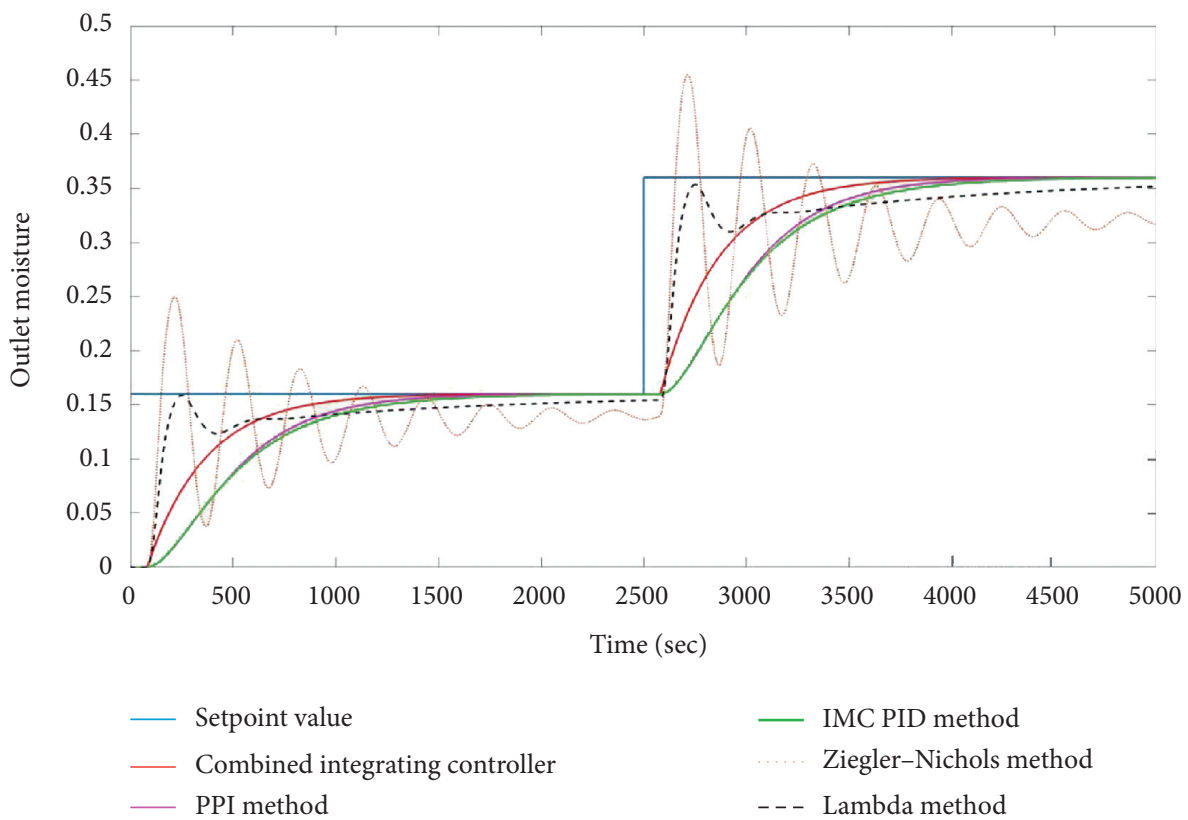

FIGURE 13: Setpoint tracking of five control algorithms under a nominal condition.

TABLE 3: The error performance index ITAE of controllers in case 1.

\begin{tabular}{lcc}
\hline ITAE & $0-2500 \mathrm{~s}$ & $2500 \mathrm{~s}-5000 \mathrm{~s}$ \\
\hline CIC & $1.725 e+04$ & $2.048 e+05$ \\
PPI & $3.290 e+04$ & $3.141 e+05$ \\
IMC PID & $3.770 e+04$ & $3.328 e+05$ \\
\hline
\end{tabular}

reached the setpoint value within the first 2000 seconds, while the Ziegler-Nichols and Lambda control algorithms failed to track the setpoint value. At $t=2000 \mathrm{~s}$, a positive disturbance and a negative disturbance are added. The five control algorithms actively suppress the disturbance and retrack the setpoint value. After adding disturbance to the Ziegler-Nichols and Lambda control algorithms, the control performance has been improved, but there are still steadystate errors. Table 4 summarizes the system error performance index ITAE of three effective control algorithms in positive disturbance and negative disturbance.

The CIC control algorithm proposed in this paper is superior to PPI and IMC in terms of time-domain dynamic performance index and steady-state performance index (perturbation amount, recovery time, and steady-state error) that suppress disturbance response. The system error performance index ITAE in Table 4 confirms that the CIC control performance is 1.5 times higher than PPI and IMC. The Ziegler-Nichols and Lambda control performance have been improved, indicating that the disturbance does not always deteriorate the control performance. The above example proves that the control performance can be improved.

8.3. Case 3. There is model adaptation when the system gets a wrong model due to data acquisition error or sensor equipment damage. Here, we assume that there is no mismatch in the system's disturbance model and that the process model of the system changes from FOPDT model to the combined integrating system model, which is a large time-delay system; that is, $\theta_{1}=200, \theta_{2}=50.3$, $G(s)=0.01 /(285.7 s+1)(s+1)$.

When the system model does not match, the ZieglerNichols and Lambda control algorithms cannot stabilize the system; from the setpoint value tracking response of Figure 15, we can see that the CIC, PPI, and IMC PID control 


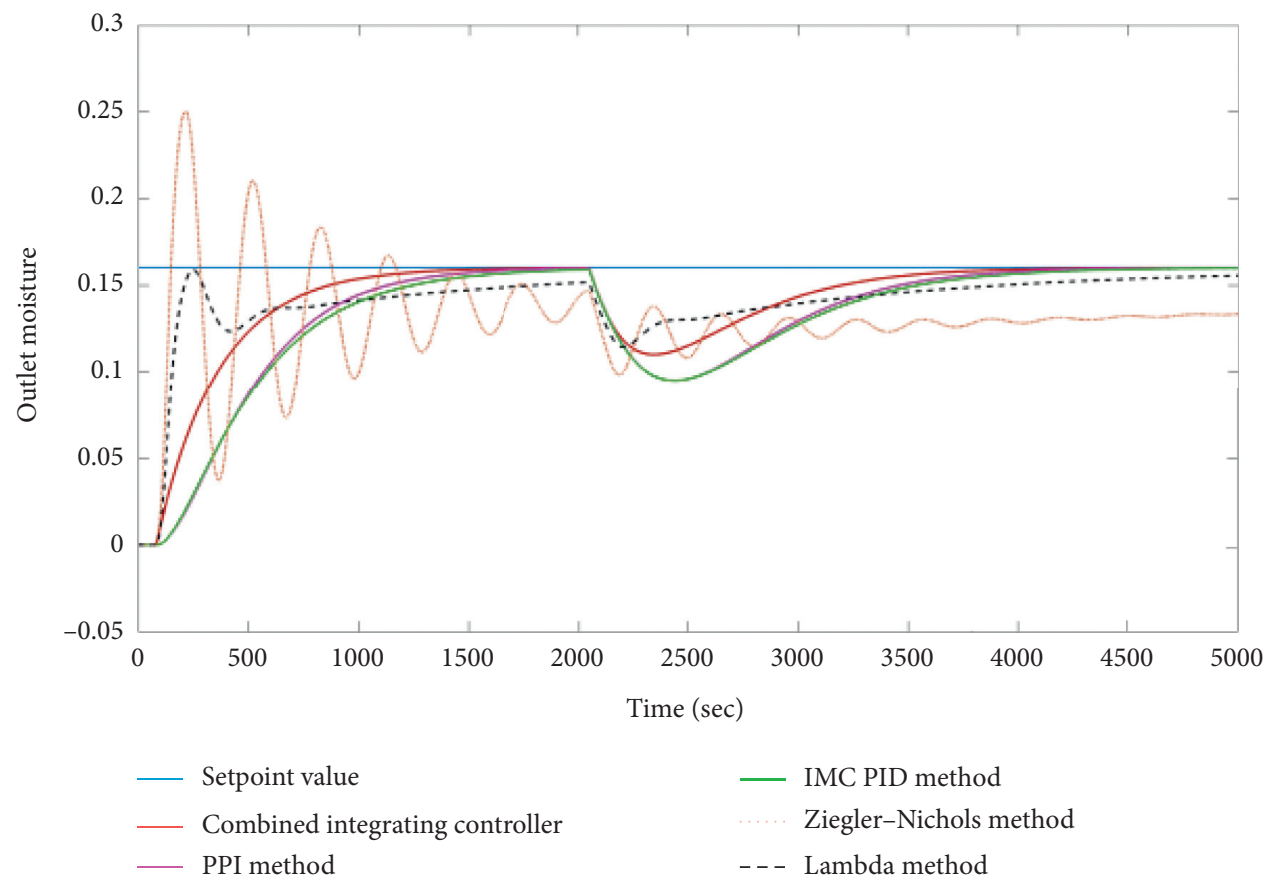

(a)

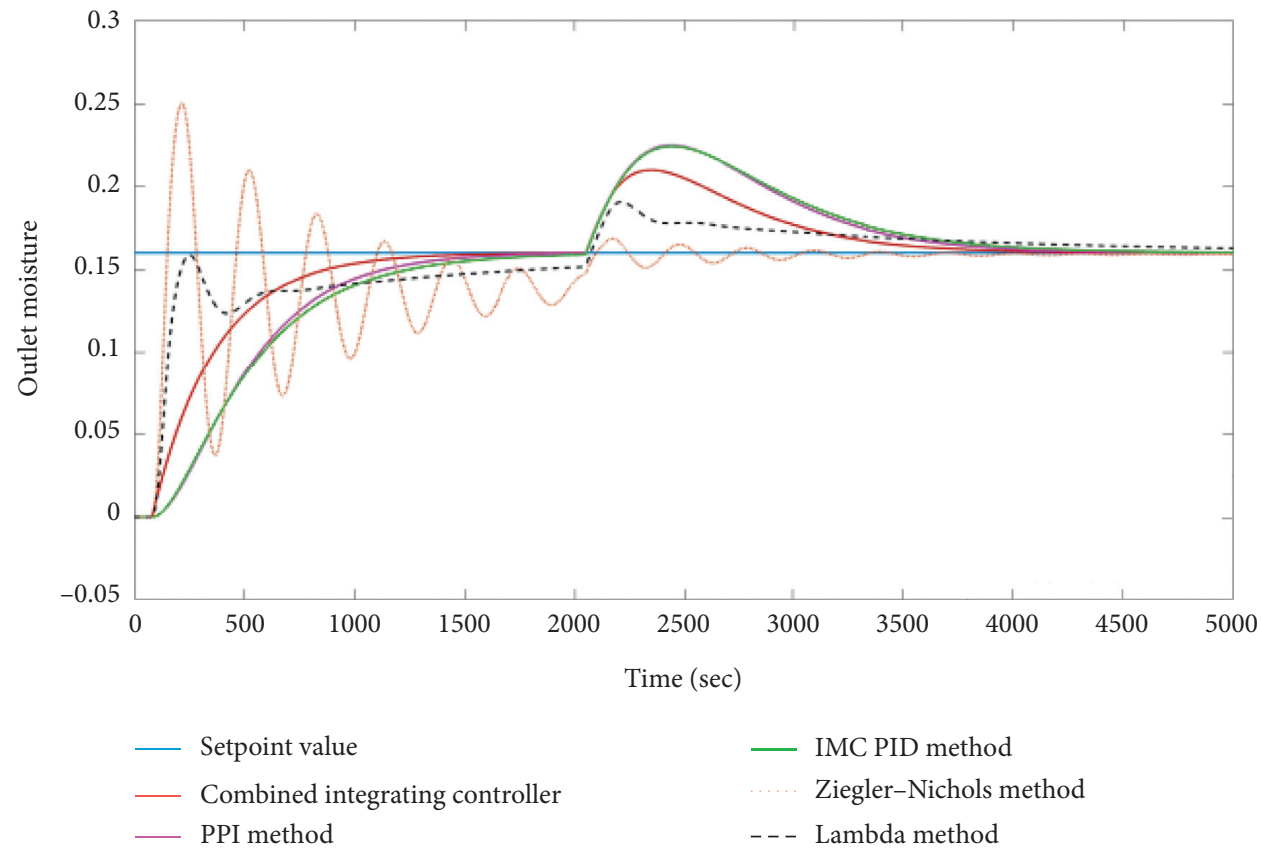

(b)

FIGURE 14: Control performance of case 2. (a) Setpoint tracking of five control algorithms under positive disturbance. (b) Setpoint tracking of five control algorithms under negative disturbance.

TABLE 4: The error performance index ITAE of controllers in case 3.

\begin{tabular}{lcccc}
\hline & \multicolumn{2}{c}{ Positive disturbance } & \multicolumn{2}{c}{ Negative disturbance } \\
\hline ITAE & $0-2000 \mathrm{~s}$ & $2000-5000 \mathrm{~s}$ & $0-2000 \mathrm{~s}$ & $2000-5000 \mathrm{~s}$ \\
CIC & $1.713 e+04$ & $1.021 e+05$ & $1.713 e+04$ & $1.023 e+05$ \\
PPI & $3.261 e+04$ & $1.568 e+05$ & $3.261 e+04$ & $1.572 e+05$ \\
IMC PID & $3.692 e+04$ & $1.658 e+05$ & $3.692 e+04$ & $1.673 e+05$ \\
\hline
\end{tabular}

algorithms can all stabilize the system; the time-domain dynamic performance of the system (rise time, settling time, and overshoot) has declined. Table 5 summarizes the system error performance index ITAE of three effective control algorithms under model mismatch.

The CIC control algorithm proposed in this paper is superior to PPI and IMC in suppressing the time-domain dynamic performance index and steady-state performance 


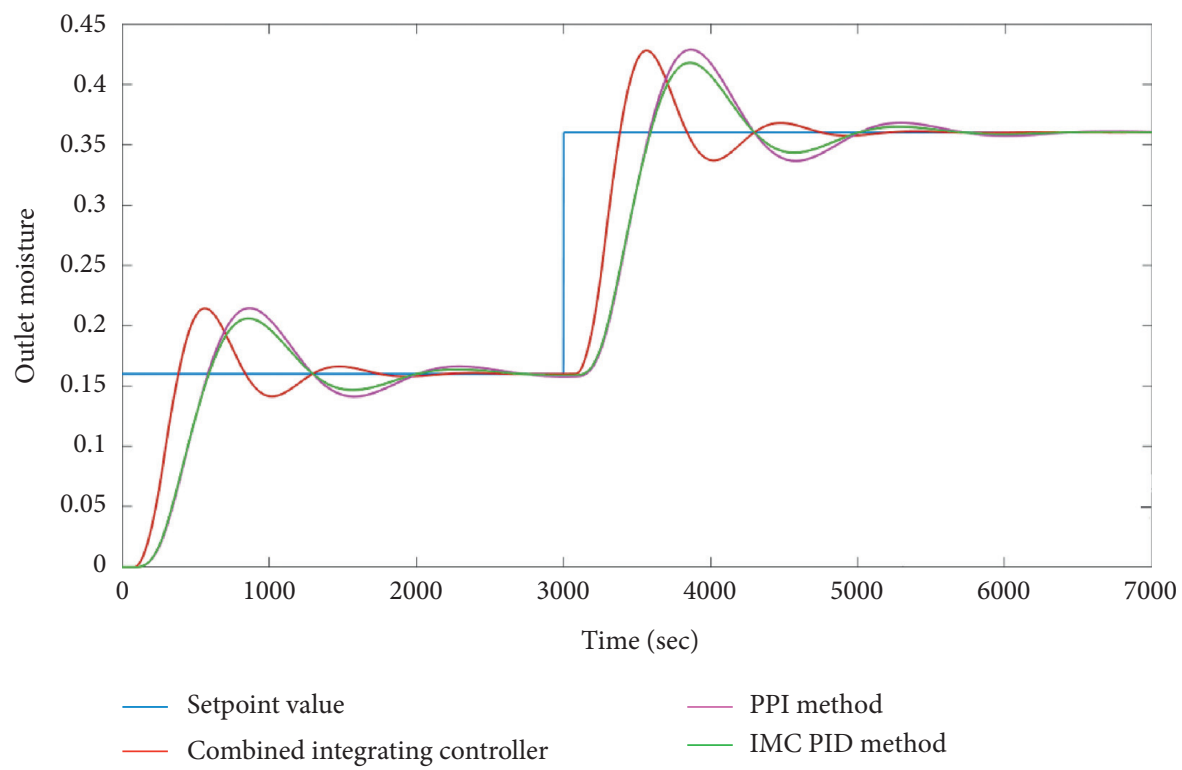

FIGURE 15: Setpoint tracking of three control algorithms under model mismatch.

TABLE 5: The error performance index ITAE of controllers in case 3.

\begin{tabular}{lcc}
\hline ITAE & $0-3000 \mathrm{~s}$ & $3000 \mathrm{~s}-7000 \mathrm{~s}$ \\
\hline CIC & $2.501 e+04$ & $2.777 e+05$ \\
PPI & $5.656 e+04$ & $4.544 e+05$ \\
IMC PID & $4.553 e+04$ & $4.046 e+05$ \\
\hline
\end{tabular}

index (rise time, settling time, and steady-state error) of the model mismatch response. ITAE from the system error performance index confirms that CIC control performance is improved two times as PPI and IMC.

The statistical data in the case analysis verifies that the CIC control algorithm proposed in this paper is superior to the traditional control algorithm in terms of time-domain dynamic performance, steady-state performance, and system error performance under setpoint tracking, disturbance suppression, and model mismatch. On the other hand, it is worth noting that modeling errors and measurement errors and disturbances do not always deteriorate the control performance. The above examples prove that the control performance can be improved.

\section{Conclusion}

This paper proposes a combined integrating controller claiming its applications and characteristics for the industry's linear time-invariant system. Extensive simulations were carried out to compare the performance of the proposed controller with popular controllers such as PID and Predictive PI control algorithms for conventional industrial processes. From the simulations, we see that the proposed controller has some superior characteristics and indexes. The proposed controller provides a more flexible framework due to the controller's output only being determined by the system input and the average value of the controller's output over a period. Further, the combined integrating term and the time-delay term are introduced into the controller structure, which breaks the disadvantage of the time-delay term. Overall, the proposed controller provides an attractive control alternative to the conventional controller.

\section{Data Availability}

The data used to support the findings of this study are available from the corresponding author upon request.

\section{Conflicts of Interest}

The authors declare no conflicts of interest.

\section{Authors' Contributions}

Angang $\mathrm{C}$ and Zhengyun $\mathrm{R}$ contributed to conceptualization, methodology, original draft preparation, and financial support. Zhiping $\mathrm{F}$ and Xue $\mathrm{F}$ contributed to validation. Angang $\mathrm{C}$ contributed to final edition check.

\section{Acknowledgments}

This research work was supported by the Fundamental Research Funds of the Central Universities (CUSF-DH-D2018100) and the China Scholarship Council (201806630067).

\section{References}

[1] Astrom, J. Karl, and H. Tore, PID Controllers: Theory, Design, and Tuning, ISA-The Instrumentation, Systems and Automation Society, Durham, NC, USA, 1995.

[2] G. Fedele, "A new method to estimate a first-order plus time delay model from step response," Journal of the Franklin Institute, vol. 346, no. 1, pp. 1-9, 2009.

[3] B. M. Sarif, D. V. A. Kumar, and M. V. G. Rao, "Comparison study of PID controller tuning using classical analytical 
methods," International Journal of Applied Engineering Research, vol. 13, pp. 5618-5625, 2018.

[4] M. Parada, R. A. Borges, D. Sbarbaro, and P. L. D. Peres, "Robust PID design for second-order processes with timedelay and structured uncertainties," IFAC Proceedings Volumes, vol. 44, no. 1, pp. 4614-4619, 2011.

[5] Q. H. Seer and J. Nandong, "Stabilization and PID tuning algorithms for second-order unstable processes with timedelays," ISA Transactions, vol. 67, pp. 233-245, 2017.

[6] M. Ajmeri and A. Ali, "Simple tuning rules for integrating processes with large time delay," Asian Journal of Control, vol. 17, no. 5, pp. 2033-2040, 2015.

[7] P. Larsson and T. Hägglund, "Comparison between robust PID and predictive PI controllers with constrained control signal noise sensitivity," IFAC Proceedings Volumes, vol. 45, no. 3, pp. 175-180, 2012.

[8] K. J. Åström and T. Hägglund, "Revisiting the Ziegler-Nichols step response method for PID control," Journal of Process Control, vol. 14, no. 6, pp. 635-650, 2004.

[9] M. R. Cesca and J. L. Marchetti, "IMC design of cascade control," Computer Aided Chemical Engineering, Elsevier, Amsterdam, The Netherlands, pp. 1243-1248, 2005.

[10] C. V. N. Rao and R. P. Sree, "IMC based controller design for integrating systems with time delay," Indian Chemical Engineer, vol. 52, no. 3, pp. 194-218, 2012.

[11] Y. Gohda, S. Masuda, and Y. Matsui, "A direct PID gain tuning based on FRIT method using optimal filter in the frequency domain," IFAC Proceedings Volumes, vol. 46, no. 11, pp. 349-354, 2009.

[12] Š. Bucz and A. Kozáková, "The sine wave tuning method: robust PID controller design in the frequency domain," Journal of Electrical Systems and Information Technology, vol. 2, no. 3, pp. 358-367, 2015.

[13] E. Grassi and K. Tsakalis, "PID controller tuning by frequency loop-shaping: application to diffusion furnace temperature control," IEEE Transactions on Control Systems Technology, vol. 8, no. 5, pp. 842-847, 2000.

[14] G. Fedele, "Independent design of multi-loop PI/PID controllers for interacting multivariable processes," Journal of Process Control, vol. 20, no. 8, pp. 922-933, 2010.

[15] J. Nandong and Z. Zang, "Multi-loop design of multi-scale controllers for multivariable processes," Journal of Process Control, vol. 24, no. 5, pp. 600-612, 2014.

[16] R. Zhang, Q. Zou, Z. Cao, and F. Gao, "Design of fractional order modeling based extended non-minimal state space MPC for temperature in an industrial electric heating furnace," Journal of Process Control, vol. 56, pp. 13-22, 2017.

[17] G. S. Virk, M. Morari, and E. Zafiriou, "Robust process control," Chemical Engineering Science, vol. 45, no. 4, p. 1149, 1990.

[18] F. Xie and H. Lu, "Delay-dependent robust H-infinity stability for uncertain discrete-time Markovian jump singular system," in Proceedings of the Chinese Control and Decision Conference, Guilin China, June 2009.

[19] L. T. Biegler, G. S. Virk, M. Morari, and E. Zafiriou, "Solution of dynamic optimization problems by successive quadratic programming and orthogonal collocation," Computers \& Chemical Engineering, vol. 8, no. 3-4, pp. 243-247, 1984.

[20] M. Cizniar, D. Salhi, M. Fikar, and E. Abderrazak, "A MATLAB package for orthogonal collocations on finite elements in dynamic optimisation," in Proceedings of the 15th International Conference Process Control, Strbske Pleso, Slovakia, June 2015.
[21] L. T. Biegler, "An overview of simultaneous strategies for dynamic optimization," Chemical Engineering and Processing: Process Intensification, vol. 46, no. 11, pp. 1043-1053, 2007.

[22] S. Kameswaran and L. T. Biegler, "Convergence rates for direct transcription of optimal control problems using collocation at Radau points," Computational Optimization and Applications, vol. 41, no. 1, pp. 81-126, 2008.

[23] J. D. Hedengren, R. A. Shishavan, K. M. Powell, and T. F. Edgar, "Nonlinear modeling, estimation and predictive control in APMonitor," Computers \& Chemical Engineering, vol. 70, pp. 133-148, 2014.

[24] D. Chen and D. E. Seborg, "PI/PID controller design based on direct synthesis and disturbance rejection," Industrial \& Engineering Chemistry Research, vol. 41, no. 19, pp. 48074822, 2002.

[25] N.-S. Pai, S.-C. Chang, and C.-T. Huang, "Tuning PI/PID controllers for integrating processes with deadtime and inverse response by simple calculations," Journal of Process Control, vol. 20, no. 6, pp. 726-733, 2010.

[26] A. A. Khan, J. R. Moyne, and D. M. Tilbury, "Favorable effect of time delays on tracking performance of type-I control systems," IET Control Theory \& Applications, vol. 2, no. 3, pp. $210-218,2008$.

[27] G. Szita and C. K. Sanathanan, "Robust design for disturbance rejection in time delay systems," Journal of the Franklin Institute, vol. 334, no. 4, pp. 611-629, 1997.

[28] W. Tan, H. J. Marquez, and T. Chen, "Performance assessment of PID controllers," Control and Intelligent Systems, vol. 32, no. 3, 2004.

[29] X.-N. Zhang and G.-H. Yang, "Performance analysis for multi-delay systems in finite frequency domains," International Journal of Robust and Nonlinear Control, vol. 22, no. 8, pp. 933-944, 2012.

[30] C. Dalen and D. Di Ruscio, "Performance optimal PI controller tuning based on integrating plus time delay models," Algorithms, vol. 11, no. 6, pp. 86-109, 2018.

[31] T. N. L. Vu and M. Lee, "Multi-loop PI controller design based on the direct synthesis for interacting multi-time delay processes," ISA Transactions, vol. 49, no. 1, pp. 79-86, 2010.

[32] D. Zheng, Z. Ren, and J.-a. Fang, "Stability analysis of multiple time-delayed system," ISA Transactions, vol. 47, no. 4, pp. 439-447, 2008.

[33] D. Zheng, Z. Ren, and J. Fang, "Stability analysis of time delayed system with coefficient uncertainty and time delay uncertainty," European Journal of Control, vol. 16, no. 1, pp. 5-13, 2010.

[34] Q. Zhang, F.-F. Lee, Y-G Wang, W. Zhang, and Q. Chen, "Performance assessment of cascade control system with nonGaussian disturbance based on minimum entropy," Symmetry, vol. 11, no. 3, pp. 379-394, 2019.

[35] O. Arrieta, R. Vilanova, and P. Balaguer, "Procedure for cascade control systems design: choice of suitable PID tunings," International Journal of Computers Communications \& Control, vol. 3, no. 3, pp. 235-248, 2008. 